\title{
Perspective: the bone-fat connection
}

\author{
Miriam A. Bredella
}

Published online: 15 May 2010

(C) ISS 2010

Bone strength depends on bone mineral density (BMD) and bone quality [1]. While BMD is typically the single best predictor of trabecular bone strength, other factors, such as bone microarchitecture or composition of bone marrow, play important roles in the determination of ultimate bone strength. In this regard attention has been focused on bone marrow fat and its role in bone strength and fracture risk. Bone marrow fat negatively affects the biomechanical strength of bone as fatty marrow is a weaker biomechanical support medium than hematopoietic marrow. Higher vertebral marrow fat content has been found in subjects with morphologic evidence of bone weakness, such as endplate depression, or compression fractures, and age-related bone loss is associated with progressive infiltration of bone marrow by fat $[2,3]$.

Recent advances in the understanding of the bone-fat connection suggest that bone marrow fat could play an important role as a diagnostic marker and a potential target for the treatment of osteoporosis. Bone and fat cells arise from the same stem cell precursor within bone marrow, and, depending on the interaction of molecular, chemical, and physical stimuli, these cells are capable of differentiation into osteoblasts or adipocytes $[4,5]$. Several studies suggest that stem cell differentiation is a mutually exclusive process with commitment to either the fat or bone lineage. This has been supported by studies in the elderly, where bone loss is associated with increase in bone marrow fat content, suggesting a preferential differentiation of stem cells into

\footnotetext{
M. A. Bredella $(\square)$

Musculoskeletal Radiology, Department of Radiology,

Massachusetts General Hospital,

Yawkey 6E, 55 Fruit Street,

Boston, MA 02114, USA

e-mail: mbredella@partners.org
}

marrow fat at the expense of bone-forming osteoblasts [6]. However, recent studies suggest that marrow adipocytes and osteoblasts can coexist. For example, in puberty both marrow fat differentiation and osteoblast differentiation are increased, which argues against a mutually exclusive process. In addition, independent pre-osteoblast and preadipocyte populations of stem cells have been shown to coexist providing a possible explanation for states of simultaneous bone and fat formation [7]. What ultimately determines the fate of the bone marrow stem cells is not fully understood and is the subject of ongoing investigation.

Several factors have been shown to influence the mesenchymal stem cell into either bone or fat cells. Estrogen has been shown to increase bone formation with associated inhibition of fat formation [8]. The peroxisomal proliferator-activated receptor gamma (PPAR- $\gamma$ ) plays an important role in stimulating fat formation within bone marrow, and activation of PPAR- $\gamma$ favors differentiation of stem cells into adipocytes rather than osteoblasts [9]. PPAR- $\gamma$ insufficiency leads to increased bone formation and higher trabecular volume [10]. In fact, increased fracture risk associated with several clinical conditions might have its origin in the bone-fat connection. PPAR- $\gamma$ agonists, such as the thiazolidinedione class of anti-diabetes drugs, which includes the drugs rosiglitazone (Avandia) and pioglitazone (Actos), cause bone loss and increased fracture risk by shifting stem cell differentiation into the fat lineage at the expense of bone formation [9]. Glucocorticoids cause osteoporosis by enhancing marrow fat formation at the expense of osteoblast differentiation [11]. Skeletal inactivity and immobilization are associated with fatty infiltration of bone marrow and conversion of stem cells into adipocytes leading to reduced bone formation. These observations raise the question of whether fatty infiltration in bone marrow is the cause of reduced bone formation or 
whether it is just filling an empty space created by the reduction in bone formation.

Bone marrow fat content also affects the assessment of $\mathrm{BMD}$, an important determinant of fracture risk. A study assessing the effects of bone marrow fat on dual-energy X-ray absorptiometry (DXA) and single-energy quantitative computed tomography (QCT) measurements of the femoral neck showed that DXA and QCT underestimate bone mineral content due to marrow fat [12].

While the analysis of bone marrow fat previously required invasive bone biopsies, we now can quantify bone marrow fat non-invasively using proton magnetic resonance spectroscopy (1H-MRS). 1H-MRS allows true quantification of bone marrow fat of the entire skeleton by calculating the lipid-towater ratio of spectra. Studies have shown that bone marrow fat content measured by $1 \mathrm{H}-\mathrm{MRS}$ in combination with BMD is of significance in evaluating skeletal integrity, more valuable than either parameter alone $[3,13]$. Therefore, $1 \mathrm{H}-$ MRS could potentially be used as a powerful extension of diagnostic MRI. Patients undergoing diagnostic MRI of the spine could be screened simultaneously for bone marrow fat to potentially assess fracture risk.

We have used 1H-MRS of bone marrow to gain further insights into the bone-fat connection and stem cell differentiation by studying abnormalities in fat metabolism in clinical states of under- and overnutrition such as anorexia nervosa and obesity.

In anorexia nervosa, a state of low subcutaneous and visceral fat, we have shown increased bone marrow fat of the lumbar spine and femur, compared to normal-weight controls, using 1H-MRS [14]. The degree of bone marrow fat was inversely associated with BMD at multiple skeletal sites measured by DXA. Interestingly, although there was a trend toward a negative correlation, lumbar vertebral bone marrow fat content did not significantly correlate with abdominal and thigh fat mass, whereas bone marrow fat content in the femoral metaphysis and diaphysis did demonstrate a significant inverse correlation with abdominal fat depots. This suggests there may be greater accumulation of fat cells within trabecular bone compartments, sites that remodel more rapidly, than cortical bone. These data also suggest that the role of marrow fat is distinct from that of subcutaneous and visceral fat depots. Our observed paradoxical increase in marrow fat when subcutaneous and visceral fat were markedly reduced raises the important question of whether impaired bone formation causes a shift into the fat lineage within bone marrow.

What about bone marrow fat in obesity? Obesity is a major public health problem, and visceral adiposity is associated with increased metabolic and cardiovascular risk. However, a longstanding concept has been that obese women are at decreased risk for developing osteoporosis, and that increased body weight protects against bone loss via mechanical loading and other factors. In contrast, recent studies have suggested that visceral adiposity and the metabolic syndrome have potential damaging effects on bone health $[15,16]$. Obese women have been found to have lower rates of bone formation, as measured by type I collagen, suggesting that increased body fat suppresses new collagen formation [17]. Using 1H-MRS we have found increased bone marrow fat in premenopausal women with high visceral fat compared to women with low visceral fat despite normal BMD. In addition, visceral fat correlated with vertebral bone marrow fat, but there was no association between bone marrow fat and body mass index (BMI), subcutaneous or total abdominal fat depots [18]. This suggests distinct metabolic roles for visceral and subcutaneous fat. Our observed correlation between visceral fat and bone marrow adiposity may provide clues as to the underlying mechanism for the negative effects of visceral fat on bone health.

In summary, there is evidence of a complex interaction between bone and fat. Stem cell differentiation into the bone or fat lineage influences skeletal homeostasis and skeletal fragility; furthermore several secondary causes of osteoporosis are associated with increased bone marrow fat. Bone marrow fat content can be quantified non-invasively using $1 \mathrm{H}-\mathrm{MRS}$ and is anticipated to be a valuable surrogate marker for the assessment of osteoporosis and fracture risk. It also allows new insights into the bone-fat connection. Shedding light into the complex interface between bone and fat and the regulation of stem cell differentiation will lead to a better understanding of several disease processes associated with bone loss and may become a target for new therapies. In the future, therapeutic manipulation of bone marrow stem cells could improve bone mass, at the expense of excess fat formation.

\section{References}

1. Osteoporosis prevention, diagnosis, and therapy. NIH Consens Statement. 2000;17:1-36.

2. Schellinger D, Lin CS, Fertikh D, et al. Normal lumbar vertebrae: anatomic, age, and sex variance in subjects at proton MR spectroscopy-initial experience. Radiology. 2000;215:910-6.

3. Schellinger D, Lin CS, Lim J, Hatipoglu HG, Pezzullo JC, Singer AJ. Bone marrow fat and bone mineral density on proton MR spectroscopy and dual-energy X-ray absorptiometry: their ratio as a new indicator of bone weakening. AJR Am J Roentgenol. 2004;183:1761-5.

4. Gimble JM, Zvonic S, Floyd ZE, Kassem M, Nuttall ME. Playing with bone and fat. J Cell Biochem. 2006;98:251-66.

5. Rosen CJ, Ackert-Bicknell C, Rodriguez JP, Pino AM. Marrow fat and the bone microenvironment: developmental, functional, and pathological implications. Crit Rev Eukaryot Gene Expr. 2009;19:109-24.

6. Rozman C, Feliu E, Berga L, Reverter JC, Climent C, Ferran MJ. Age-related variations of fat tissue fraction in normal human bone 
marrow depend both on size and number of adipocytes: a stereological study. Exp Hematol. 1989;17:34-7.

7. Post S, Abdallah BM, Bentzon JF, Kassem M. Demonstration of the presence of independent pre-osteoblastic and pre-adipocytic cell populations in bone marrow-derived mesenchymal stem cells. Bone. 2008;43:32-9.

8. Dang ZC, van Bezooijen RL, Karperien M, Papapoulos SE, Lowik CW. Exposure of KS483 cells to estrogen enhances osteogenesis and inhibits adipogenesis. J Bone Miner Res. 2002; 17:394-405.

9. Rzonca SO, Suva LJ, Gaddy D, Montague DC, Lecka-Czernik B. Bone is a target for the antidiabetic compound rosiglitazone. Endocrinology. 2004;145:401-6.

10. Akune T, Ohba S, Kamekura S, et al. PPARgamma insufficiency enhances osteogenesis through osteoblast formation from bone marrow progenitors. J Clin Invest. 2004;113:846-55.

11. Mazziotti G, Angeli A, Bilezikian JP, Canalis E, Giustina A. Glucocorticoid-induced osteoporosis: an update. Trends Endocrinol Metab. 2006;17:144-9.

12. Kuiper JW, van Kuijk C, Grashuis JL, Ederveen AG, Schutte HE. Accuracy and the influence of marrow fat on quantitative CT and dual-energy X-ray absorptiometry measurements of the femoral neck in vitro. Osteoporos Int. 1996;6:25-30.

13. Schellinger D, Lin CS, Hatipoglu HG, Fertikh D. Potential value of vertebral proton MR spectroscopy in determining bone weakness. AJNR Am J Neuroradiol. 2001;22:1620-7.

14. Bredella MA, Fazeli PK, Miller KK, et al. Increased bone marrow fat in anorexia nervosa. J Clin Endocrinol Metab. 2009;94:2129-36.

15. Gilsanz V, Chalfant J, Mo AO, Lee DC, Dorey FJ, Mittelman SD. Reciprocal relations of subcutaneous and visceral fat to bone structure and strength. J Clin Endocrinol Metab. 2009;94:3387-93.

16. von Muhlen D, Safii S, Jassal SK, Svartberg J, Barrett-Connor E. Associations between the metabolic syndrome and bone health in older men and women: the Rancho Bernardo Study. Osteoporos Int. 2007; 18:1337-44.

17. Papakitsou EF, Margioris AN, Dretakis KE, et al. Body mass index (BMI) and parameters of bone formation and resorption in postmenopausal women. Maturitas. 2004;47:185-93.

18. Bredella MA, Torriani M, Hosseini Ghomi R, et al. Vertebral bone marrow fat is positively associated with visceral fat and inversely associated with IGF-1 in obese women. Obesity. 2010 (in press). 